\title{
Monoamine oxidase in pancreatic islets, exocrine pancreas, and liver from rats. Characterization with clorgyline, deprenyl, pargyline, tranylcypromine, and amezinium*
}

\section{S. Lenzen, H. Nahrstedt, and U. Panten}

Institut für Pharmakologie und Toxikologie, Universität Göttingen, Robert-Koch-Strasse 40, D-3400 Göttingen, Federal Republic of Germany

Naunyn-Schmiedeberg's Arch Pharmacol (1983) 324:190-195

On page 191, line 17 of the right-hand column should read:

Phenylethylamine was preferentially deaminated by MAO 